\title{
Impact of gravity on the flow pattern in a locally heated two-layer system
}

\author{
V. B. Bekezhanova ${ }^{1,2}$. O. N. Goncharova ${ }^{3}$
}

Received: date / Accepted: date

\begin{abstract}
Problem of thermocapillary convection is 26 studied to analyze peculiarities of the flows arising in 27 a gas-liquid system under action of an intense local thermal exposure. The "stream function - vorticity" formulation of the Navier - Stokes equations in the Boussi- ${ }_{28}$ nesq approximation are used to describe the fluid flows.

The kinematic and dynamic conditions on the free boun- ${ }_{29}$ dary are stated in terms of tangential and normal ve- ${ }_{30}$ locities, while temperature conditions at the lower or ${ }_{31}$ upper boundary of the system take into account the ${ }_{32}$ presence of point heaters. Special attention is given to t3 $_{33}$ the study of the influence of the gravity intensity on ${ }_{34}$ the dynamics of heat and mass transfer in fluid layers ${ }_{35}$ and character of the interface deformations. Theoret- ${ }_{36}$ ical study of the thermocapillary convection includes ${ }_{37}$ development of the mathematical model and effective ${ }_{38}$ numerical algorithm. The results of numerical study of ${ }_{39}$ structure and nature of convective flows in the cavity 40 being in the terrestrial or microgravity conditions and ${ }_{41}$ of the evolution of the interface allow one to validate ${ }_{42}$ the developed mathematical model, and to specify dom- ${ }_{43}$ inant mechanisms determining the flow regimes.
\end{abstract}

Keywords Thermocapillary convection · Free bound- ${ }_{46}$ ary $\cdot$ Local heating $\cdot$ Numerical simulation

PACS 02.60.Cb - 05.60.-k · 02.70.-c

${ }^{1}$ Department of Differential Equations of Mechanics, Institute of Computational Modelling SB RAS, 660036, Akadem- ${ }^{51}$ gorodok, 50/44, Krasnoyarsk, Russia

E-mail: vbek@icm.krasn.ru .

2 Institute of Mathematics and Computer Science, Siberian Federal University, 660041, Svobodny, 79, Krasnoyarsk, Russia

${ }^{3}$ Altai State University, 656049, pr Lenina 61, Barnaul, Rus- 56 sia

E-mail: gon@math.asu.ru
Mathematics Subject Classification (2000) MSC $76 \mathrm{~T} 10 \cdot 76 \mathrm{R} 99$

\section{Introduction}

The range of problems associated with convective flows has been significantly expanded in the last decades. This tendency is occasioned by the new applications of the fluidic system in different technologies. Development of space research leads to a revision of many theoretical approaches, which found experimental and practical confirmation by study of the convection problems in the terrestrial conditions. The adjoint problems of thermal gravitational and thermocapillary convection occurring in the non-Boussinesq conditions need new approaches, more complicated statements and investigation methods in order to take into account governing factors and to determine main and secondary mechanisms of the flows. Modern mathematical models to describe the convection in microscales, weak gravitational and fast-variable temperature fields have been derived in [1] (see also the references therein [1]).

Even without phenomena of phase transition the mathematical modeling of dynamics of various heatand mass transfer processes remains rather difficult. The careful study of the problems of the thermal gravitational and thermocapillary convection in the fluids, of interfacial processes, of coupling of convection in the liquids with interfacial phenomena is presented in [27]. Particular attention is always paid to formulation of the interface conditions between two moving media, to determination of an interfacial area (interfacial boundary), and to mathematical idealization of this area (or this surface). Any method of interface determination should guarantee fulfillment of the laws of mass, mo- 
mentum and energy conservation, which are the basis of 54 many mathematical theories for fluid flow description. 55 Rigorous and detailed derivation of the free boundary 56 conditions can be found in $[8,9]$ for stationary and non- 57 stationary problems. They are based on the assumption 58 that the interface is a moving smooth material surface 59 similar to the image of a fixed surface in the space of 60 Lagrangian coordinates defined by the liquid motion. In 61 the usual free boundary problem statements these con- 62 ditions are working excluding mass transport through 63 the interface. Derivation of the dynamic condition is 64 based on the law of momentum conservation. The rela- 65 tion sets a balance of the bulk and surface forces; the 66 latter forces act on the boundary of the contact region 67 of two fluids in a material volume. The different forms 68 of the energetic conditions describe an energy trans- 69 fer across the interface. They are based on the law of 70 total energy conservation. In [1] the derivation of the 71 free boundary conditions are explained in depth, using 72 the appropriate formulae of differential geometry and 73 the relations between classical translation formula and 74 its surface analogue. The hypotheses allows one to de- 75 scribe motion of a viscous incompressible liquid with 76 interfaces or free surfaces by the Navier-Stokes equa- 77 tions or their Oberbeck - Boussinesq approximation. It 78 should be noted that use of the equations is often fairly 79 adequate even as the interfaces becomes unstable and 80 changes in the interface topology take place, provided 81 that fluids remain to be immiscible. However, use of the 82 Navier - Stokes equations or their approximations is not 83 fully defensible in case of the development of secondary 84 instability of the interface and a decrease in character- 85 istic scales of the flow. A rational description of motion ${ }_{86}$ of similar system should be given in terms of mechan- ${ }_{87}$ ics of heterogeneous media. The additional continuity ${ }_{88}$ conditions postulated on the interface, use of thermodynamical laws and some further assumptions of the inter- ${ }_{90}$ face thermodynamics have been also discussed therein ${ }_{91}$ [1]. Theoretical study of the problem of convection in ${ }_{92}$ a two-layer system with the finding of deformable in- ${ }_{93}$ terface position is extremely difficult. Without dwelling ${ }_{94}$ any more on problems in the non-stationary statements ${ }_{95}$ including the dynamic contact angle question, we turn ${ }_{96}$ to the numerical research based on those mathematical ${ }_{97}$ models of fluid flows with an interface, in which this ${ }_{98}$ question is correctly solved (see, for instance, [10,11]). ${ }_{99}$

Specific substantial point is determination of a real100 interface or free boundary position, that evidently is ${ }^{101}$ solved with the help of the interface conditions. We102 note that the real interface position can be obtainedio3 as a result of numerical investigation of the stability of 104 flows computed in a domain with fixed boundaries. $\mathrm{In}_{105}$ this case the system of the equations for "main flow" perturbations and of the dynamic or kinematic interface condition, as an interface equation, are used. In the frame of original problem the question of interface position finding arises most acutely when using the finite-difference methods in computations of flows in the domains with interfaces. We do not concern numerical studies on this subject that are carried out within the framework of the thin layer approximation. In the context of the Oberbeck - Boussinesq model of convection the thermocapillary flows caused by action of the thermal point sources in a two-layer system were investigated [12]. The structure of the temperature and velocity fields and dynamics of changing the thermocapillary interface topology were calculated with the help of the original numerical method $[13,14]$. This method includes (i) formulation of the problem in terms of stream function and vorticity; (ii) transition to new variables (or straight line procedure); (iii) the stabilizing-correction finite-difference scheme characterized by full approximation of equation on the fractional step and, with it, by correction procedure with the aim of improving stability on second step; (iv) finding of the interface position at all the time moments with use of the kinematic conditions; (v) determination of normal and tangential velocities at the interface points. In [10] the computational algorithm to calculate the free boundary position at any time steps has been described in details. In [10] and [11] the "stream function-vorticity" formulation of the problem is performed also. But in comparison with $[13,14]$, the dynamic interface condition, that is the normal stress balance condition, is used to compute the interface position.

The heightened interest to the problems of convection under the phase transition at interfaces is caused by ground and space experiments $[15,16]$. In [15] the results of a systematic experimental study of the complex thermal patterns corresponding to the coupling of interfacial effects induced by evaporation, thermocapillary forces and shear flow were presented. Physical experiments [16] were carried out to study the liquid dynamics in the horizontal layers or cavities, the processes on the liquid-gas interface of the limited size under co-current dry or wet gas flows. They allowed us to obtain data of quantitative measurements of average velocities of vortex structures in the liquids, of the surface temperature and temperature gradients, and of the characteristics of the interface movements caused by the gas flows.

Experimental study of breakdown of the thin horizontal layers of ethanol and water by a local heating from the substrate has been performed in [17]. The basic stages of process of the liquid layer rupture has been determined and the time of a dry spot forma- 
tion has been measured. Thermocapillary deformations and breakdown of a thin layer of viscous incompressible volatile liquid with a free surface have been modelled on the basis of lubrication approximation [18]. Numerical algorithm for the joint solving the equations of energy and layer thickness evolution has been developed and free surface deformations have been calculated with use of the finite volume method.

New experiments [19] are devoted to study the flows arising in a two-layer system due to a laser beam heating on the free boundary and to investigations of the deformable surface behavior. Profiles of the thermocapillary deformations of both internal interface and free ${ }_{50}$ surface were measured. Time of appearing the stationary rupture of the upper layer as well as time of full ${ }_{51}$ relaxation of the gap ("healing" of the layer by turning ${ }_{52}$ off the pump laser) were ascertained depending on the ${ }_{53}$ upper layer thickness for different types of working liq- ${ }_{54}$ uids. Dependence of the rupture diameter on the ther- ${ }_{55}$ mal load intensity was obtained. To study exhaustively 56 mechanisms determining the dynamics of observed pro- ${ }_{57}$ cesses in the two-layer systems under conditions of lo- ${ }_{58}$ cal heating or/and phase transfer at the interfaces one ${ }_{59}$ needs to perform a mathematical modeling of the inter- 60 acted processes. The main points are the choice of basic ${ }_{61}$ model (it implies form of governing equations) and for- ${ }_{62}$ mulation of the interface conditions. The correct mathe- ${ }_{63}$ matical statement of the investigated problems enables to develop rather fine computational methods, which will give one a possibility to calculate the flow patterns and real position of the interfaces.
In the present paper we develop the mathematical model of the thermocapillary convection in a twolayer system of fluids bounded by rigid walls. The local thermal load is applied to the system from below 64 or above. The original model is based on the Navier -65 Stokes equations in the Oberbeck - Boussinesq approxi- 66 mation. A variant of the numerical algorithm described 67 in $[13,14]$ is elaborated. It allows one to find correctly 68 the shape of the internal deformable interface having 69 parts with strongly changing curvature. A series of cal- 70 culations for the case of one heater centrally arranged $7_{11}$ on the lower or upper wall of the cuvette was performed. 72 Typical patterns of velocity and temperature fields in 73 the nitrogen - ethanol system being in the terrestrial or 74 microgravity conditions are presented. The position of 75 the interface for all considered cases is calculated, and 76 character of the interface deformations is analyzed. $\quad 77$

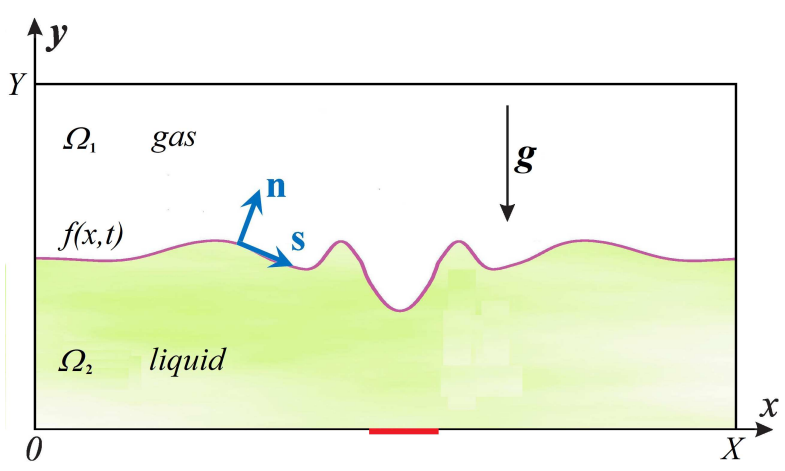

Fig. 1 Geometrical configuration of the two-layer system

\section{Mathematical model}

2.1 Governing equations and general parameters

The problem of convection in a system of two immiscible fluids (liquid and gas) with a common thermocapillary interface is studied under assumption that both media are the viscous incompressible liquids. The twolayer system fill a cavity with solid impermeable walls. Mathematical model for description of the thermocapillary flows includes the Navier-Stokes equations in the Oberbeck - Boussinesq approximation, the initial conditions determining the initial state of the two-layer system, boundary conditions on the external boundaries of the cell and matching conditions on the internal interface. One of the relations on the surface should provide a finding the interface position at each time step.

Let the Cartesian coordinate system be chosen so that the gravity acceleration vector $\mathbf{g}$ is directed opposite to the $O y$ axis $(\mathbf{g}=-g \mathbf{i}, \mathbf{i}$ is the unit vector of the $O y$ axis). Two viscous incompressible liquids fulfill a rectangular domain $\Omega$ with boundary $\partial \Omega$. Regions $\Omega_{1}$ and $\Omega_{2}$

$$
\begin{gathered}
\left.\Omega_{1}=\{(x, y): 0<x<X, f(x, t)<y<Y)\right\}, \\
\Omega_{2}=\{(x, y): 0<x<X, 0<y<f(x, t)\}
\end{gathered}
$$

are the parts of $\Omega$, and they are filled by gas and liquid, respectively (see (Fig. 1)). The domains are separated by the thermocapillary interface $\Gamma$ defined by equation $y=f(x, t)$. The external boundaries of the cuvette are rigid walls, so that corresponding parts of $\partial \Omega$ that confine domains $\Omega_{1}$ and $\Omega_{2}$ are defined by sets of the rectilinear segments: $\partial \Omega_{1}=\{x=0, x=X, y=Y\}$ and $\partial \Omega_{2}=\{x=0, x=X, y=0\}$.

Let the upper layer thickness $h_{1}$ at the initial instant $t=0$ be chosen for the characteristic length. The characteristic values of velocity and temperature are denoted by $u_{*}$ and $T_{*}$. The characteristic value for pressure was chosen equal to $\rho_{2} u_{*}^{2}$. To describe convective motion of $j$-th medium the Oberbeck-Boussinesq 
approximation of the Navier - Stokes equations is used. 42 In the non-dimensional form the governing equations ${ }_{43}$ are written as follows:

$$
\begin{aligned}
& \partial_{t} \mathbf{v}_{j}+\left(\mathbf{v}_{j} \cdot \nabla\right) \mathbf{v}_{j}= \\
& =-\nabla p_{j}+\operatorname{Re}_{j}^{-1} \Delta \mathbf{v}_{j}-\operatorname{Gr}_{j} \operatorname{Re}_{j}^{-2} T_{j} \mathbf{i}
\end{aligned}
$$

$\operatorname{div} \mathbf{v}_{j}=0$,

$\partial_{t} T_{j}+\mathbf{v}_{j} \cdot \nabla T_{j}=\operatorname{Pr}_{\mathrm{j}}^{-1} \operatorname{Re}_{\mathrm{j}}^{-1} \Delta T_{j}$,

Here and thereinafter the indexes $j=1,2$ are related ${ }^{50}$ to the upper $(j=1)$ and lower $(j=2)$ fluids, respec- 51 tively; $\mathbf{v}_{j}=\left(u_{j}, v_{j}\right)$ is the velocity vector, $T_{j}$ is the tem- 52 perature, $p_{j}$ is the pressure (deviation of pressure from ${ }^{53}$ the hydrostatic one). Following parameters arise in the ${ }_{54}$ transition to the dimensionless problem statement: the Reynolds number $\operatorname{Re}_{j}=u_{*} h_{1} / \nu_{j}$, the Prandtl number ${ }_{56}^{55}$ $\operatorname{Pr}_{\mathrm{j}}=\nu_{j} / \chi_{j}$, the Grashof number $\mathrm{Gr}=\beta_{j} T_{*} g h_{1}^{3} / \nu_{j}^{2}{ }_{57}$ Here $\nu_{j}, \chi_{j}, \beta_{j}$ are the kinematic viscosity, thermal diffusivity and thermal expansion coefficients of the fluids, ${ }^{58}$ respectively. The previous notations for time and spa- 59 tial variables, their variation ranges, and also interface 60 equation are kept unchanged.

\subsection{Conditions on the interface}

Consider the surface $\Gamma=\{(t, x, y): y=f(x, t)\}$ be- ${ }^{63}$ tween the upper $\Omega_{1}$ and lower $\Omega_{2}$ layers. We assume ${ }^{64}$ that $\Gamma$ is a thermocapillary interface, along which the 65 tangent forces act. At this the surface tension coefficient ${ }_{66}$ $\sigma(T)$ linearly depends on temperature $\sigma(T)=\sigma_{0}-{ }_{67}$ $\sigma_{T}\left(T-T_{0}\right)$, where $\sigma_{0}, \sigma_{T}$ are given positive constants, $T_{0}$ is a reference temperature. The dimensionless form ${ }^{68}$ of the relation for $\sigma$ is given by $\sigma(T)=1-\mathrm{MaCa}\left(T-T_{0}\right)^{69}$ with the Marangoni number Ma $=\left(\sigma_{T} T_{*}\right) /\left(\rho_{2} u_{*} \nu_{2}\right)^{70}$ and the capillary number $\mathrm{Ca}=\rho_{2} u_{*} \nu_{2} / \sigma_{0}$.

To formulate the interface conditions we introduce the unit tangent and normal vectors on the interface ${ }^{72}$

$$
\begin{aligned}
& \mathbf{s}=\left(1 / \sqrt{1+\partial_{x}^{2} f}, \partial_{x} f / \sqrt{1+\partial_{x}^{2} f}\right) \\
& \mathbf{n}=\left(-\partial_{x} f / \sqrt{1+\partial_{x}^{2} f}, 1 / \sqrt{1+\partial_{x}^{2} f}\right)
\end{aligned}
$$
lower liquid $\mathbf{n}$ is the unit vector of the outer normal to the interface $\Gamma$. Then, the velocity of points lying on the interface can be presented in the form $\mathbf{V}={ }_{79}^{78}$ $v_{n} \mathbf{n}+v_{s} \mathbf{s}$, where $v_{n}, v_{s}$ are the normal and tangent ${ }_{80}^{79}$ components of the velocity vector. We postulate in the ${ }^{80}$ proposed mathematical model, that velocities of fluid ${ }_{82}^{81}$ particles for both liquids on $\Gamma$ are the same.

The interface conditions based on the conservation laws and some additional assumptions $[1,7]$ have the ${ }^{84}$ following dimensionless form:

$\left(v_{2}\right)_{n}=\left(v_{1}\right)_{n}=V, \quad V=-\partial_{t} f / \sqrt{1+\partial_{x}^{2} f}$, $-p_{2}+2 \operatorname{Re}_{2}^{-1} \mathbf{n} \cdot \mathbf{D}\left(\mathbf{v}_{2}\right) \mathbf{n}=-p_{1}+2 \bar{\rho} \operatorname{Re}_{1}^{-1} \mathbf{n} \cdot \mathbf{D}\left(\mathbf{v}_{1}\right) \mathbf{n}+$ $+2 \mathrm{Ca}^{-1} \mathrm{Re}_{2}^{-1} \sigma H$,

$2 \mathbf{s} \cdot \mathbf{D}\left(\mathbf{v}_{2}\right) \mathbf{n}-2 \bar{\rho} \bar{\nu} \mathbf{s} \cdot \mathbf{D}\left(\mathbf{v}_{1}\right) \mathbf{n}=-\mathrm{Ma}_{s} T$,

where $\left(v_{j}\right)_{n}=\mathbf{v}_{j} \cdot \mathbf{n}$ are the normal components of the velocity vectors $\mathbf{v}_{j}(j=1,2), V$ is the interface velocity in the normal direction, $\mathbf{D}(\mathbf{v})$ is the stress tensor, $\bar{\rho}=$ $\rho_{1} / \rho_{2}, \bar{\nu}=\nu_{1} / \nu_{2}$ are the ratios of fluids densities and kinematic viscosities.

The additional interface condition follows from the assumption about velocity continuity at the interface $\Gamma$ and leads to the equality of the tangential velocities $\left(v_{j}\right)_{s}(j=1,2)$ :

$\left(v_{1}\right)_{s}=\left(v_{2}\right)_{s}$.

The temperature conditions at the interface $\Gamma$ is written as continuity conditions of temperature and heat fluxes

$T_{1}=T_{2}, \quad \partial_{n} T_{2}-\bar{\kappa} \partial_{n} T_{1}=0$,

where $\bar{\kappa}=\kappa_{1} / \kappa_{2}$ is the ratio of fluids thermal conductivities.

\subsection{Boundary conditions at cavity walls}

The boundary conditions for the velocity functions at solid surfaces $x=0, x=X, y=0, y=Y$ correspond to no-slip condition for viscous fluids:

$\left.\mathbf{v}_{j}\right|_{\partial \Omega_{j}}=0$.

Boundary conditions of the first kind for the temperature function are set at the lateral walls:

$\left.T_{j}\right|_{x=0}=0,\left.\quad T_{j}\right|_{x=X}=0$.

Conditions for the temperature at the lower and upper boundary take into account the presence of local heater. In general form the conditions are written as follows:

$$
\begin{gathered}
\left.T_{1}\right|_{y=Y, x \notin H^{u p}}=0,\left.\quad T_{1}\right|_{y=Y, x \in H^{u p}}=\theta^{u p}(t), \\
\left.T_{2}\right|_{y=0, x \notin H^{s}}=0,\left.\quad T_{2}\right|_{y=0, x \in H^{s}}=\theta^{s}(t),
\end{gathered}
$$

where $H$ is the area occupied by heater, $\theta$ is the temperature of the heater. When placing heater on a substrate $H^{u p}=\oslash$ and $\theta^{u p}(t)=0$, and the temperature $T_{1}=0$ is maintained on the entire upper wall. If the thermal source is arranged from above, then $H^{s}=\oslash$ and $\theta^{s}(t)=0$, i.e. the temperature of the lower boundary is constant everywhere equal to $T_{2}=0$. Areas $H$ and values $\theta$ in conditions (2.11) are determined depending on the requirements of specific problems. In the present work we consider case of centrally arranged heater on the lower or upper wall with commutated mode of heating, when the heater temperature can be changed abruptly. Size of heater and thermal regime are given in the corresponding sections along with the results of numerical calculations. 
39 $F_{1}=$ Ma $\partial_{s} \theta+2(1-\bar{\rho} \bar{\nu})\left(\partial_{s} v_{n}+v_{s} R^{-1}\right)$,

${ }_{40} \quad F_{2}(t, x)=-2\left[\partial_{s}\left(\partial_{n}\left(v_{2}\right)_{n}-\bar{\rho} \bar{\nu} \partial_{n}\left(v_{1}\right)_{n}\right)\right]+$

${ }_{41}+2\left[(1-\bar{\rho} \bar{\nu}) \partial_{s}\left(\partial_{x} f v_{s} R^{-1}\right)\right]+$

${ }_{42}+\mathrm{Ca}^{-1} \partial_{s}\left[(1-\mathrm{MaCa} T) R^{-1}\right]-$

${ }_{43}-\left(\mathrm{Gr}_{2} \operatorname{Re}_{2}^{-1}-\bar{\rho} \bar{\nu} \mathrm{Gr}_{1} \operatorname{Re}_{1}^{-1}\right) T \partial_{x} f / \sqrt{1+\partial_{x}^{2} f}+$
Here $T$ is the temperature function, as before.

Relations (2.4) - (2.7) on the interface and boundary ${ }^{63}$ Upon that balance conditions (2.5) and (2.6) will be ${ }^{65}$ derived as relations for vorticity functions in the terms ${ }^{66}$ of normal and tangential velocities $v_{n}=-\partial_{s} \psi, v_{s}={ }_{67}$

Using the definition of normal velocity component ${ }^{68}$ and its connection with stream function, the kinematic ${ }^{69}$ condition (2.4) at the interface $\Gamma$ can be rewritten in ${ }^{70}$ the form:

$\partial_{t} f+\sqrt{1+\partial_{x}^{2} f} \partial_{s} \psi_{2}=0$.

The dynamic conditions $(2.5),(2.6)$ at the interface $\Gamma^{2}$ can be presented as follows:

The first equality is the analogue of the tangent com- $^{77}$ ponent of the dynamic condition, and the second one ${ }^{78}$ expresses equivalent of its normal component. Function ${ }^{79}$ $F_{1}$ takes into account the thermocapillary force action, ${ }^{80}$ and $F_{2}$ includes a contribution of pressure-jump and ${ }^{81}$ effects of the problem nonstationarity:
$+\operatorname{GaRe}_{2}(1-\bar{\rho}) \partial_{x} f / \sqrt{1+\partial_{x}^{2} f}$

$+\operatorname{Re}_{2}\left[(\bar{\rho}-1) \partial_{t} v_{s}+(\bar{\rho}-1) v_{s} \partial_{s} v_{s}+\right.$

$\left.+(1-\bar{\rho}) \partial_{x} f v_{n}^{2} R^{-1}+v_{n}\left(\omega_{2}-\bar{\rho} \omega_{1}\right)\right]$.

Here $R$ is the interface curvature radius $\left(1 / R=\partial_{x x} f /\right.$ $\left.\left(1+\partial_{x}^{2} f\right)^{3 / 2}\right), \mathrm{Ga}=g h / u_{*}^{2}$ is the Galileo number. Note that a procedure of derivation of conditions (3.6) and (3.7) implies the use of equation (3.3) supposed to be valid at interface, relation, that is a consequence of differentiation of the kinematic condition (3.5) along the interface, and conditions of equality of the tangential velocities (2.7) and their tangential derivatives on $\Gamma$. Besides, equation (2.1) scalarly multiplied by $\mathbf{n}$ was used to transform condition (2.5).

Due to the equality of the tangential velocities of fluids filling $\Omega_{1}$ and $\Omega_{2}$ at the interface $\Gamma$ (2.7) and to the volume preserving conditions for each medium, we obtain the following conditions at this interface:

$\psi_{1}=\psi_{2}, \quad \partial_{n} \psi_{2}-\partial_{n} \psi_{1}=0$

Thermal boundary regime is determined, as before, by relations $(2.10),(2.11)$.

No-slip conditions (2.9) for the velocity vectors at rigid walls $x=0, x=X, y=0, y=Y$ lead to the relations for the stream functions:

$\left.\psi_{j}\right|_{\partial \Omega_{j}}=0,\left.\quad \partial_{n} \psi_{j}\right|_{\partial \Omega_{j}}=0$.

To find values of vorticity functions $\omega_{j}$ at solid boundaries $\partial \Omega_{j}$ we will use the Tom's condition [20] after problem discretization in time and space.

\section{General scheme of solution of the coupled problem}

We restrict ourselves only to several comments relative to numerical technique. Specific elements of the computational procedure developed in $[13,14]$ are: (i) transition from the domains $\Omega_{j}$ with curvilinear boundaries to the canonic computation regions (here the squares $[0,1] \times[0,1])$ and calculations of unknown functions $\psi_{j}$, $\omega_{j}, T_{j}$ in the regions with straight limiting lines; (ii) the use of "the finite-difference scheme of stabilizing correction" (a variant of alternating direction methods $[21,22])$. This scheme is unconditionally stable one and has formally the second order of accuracy. As a final of approximation we will obtain the systems of linear algebraic equations which can be stably solved by the variants of the Gaussian elimination usual sweep method or the Thomas algorithm in the spatial variable directions.

1. We will proceed from a given state that is characterized by known distributions $\psi_{j}, \omega_{j}, T_{j}$ and position of the interface $f(t, x)$. It is considered that 
the fluids are at rest and have constant tempera- 51 ture at initial instant $t=0$; upon that, the interface ${ }_{52}$ between them is flat. With given basic character- 53 istics we solve numerically the equation (3.5) and ${ }_{54}$ find new position of $\Gamma$; thereby normal velocity $v_{n} 5$ at the interface is defined for all points lying on the 56 interface.

2. At each time step we introduce new spatial variables 58 which are connected with $x, y$ for lower fluid in $\Omega_{2} 59$ as $x=\xi, y=\eta f(\xi, t)$, and for upper fluid in $\Omega_{1} 60$ as $x=\xi, y=\eta(Y-f(\xi, t))+f(\xi, t)$. At this all 61 boundaries of computational domains, including the 62 interface, will coincide with coordinate lines of a new 63 mesh.

64

3. Sweep procedure coefficients are calculated to solve 65 the motion and heat transfer equations for both lay- 66 ers of the system.

4. Boundary conditions are determined to find hydro- 68 dynamic characteristics. For this tangential velocity 69 $v_{s}$ at $\Gamma$ is computed, then functions $F_{1}$ and $F_{2}$ can 70 be defined.

71

5. The unknown functions $T_{j}$ are found numerically 72 on the basis of equations (3.4) and boundary con- 73 ditions (2.8) at interface and (2.10), (2.11) on the 74 fixed boundaries.

6. Knowing functions $T$ and $f$ for all grid points of 76 corresponding computational domains, we solve nu- 77 merically equations (3.2) with boundary conditions 78 (3.6), (3.7) on the interface and with Tom's condi- 79 tions resulted from (3.9) on the fixed boundaries to 80 find $\omega_{j}$.

7. In each time step we introduce the iteration processes to compute problem (3.3), (3.5), (3.8), $(3.9)^{81}$ and to find the unknown functions $\psi_{j}$. Iteration process is organized with using of the convergence cri- ${ }^{82}$ teria. The velocity vector components $u_{j}, v_{j}$ can be ${ }_{84}^{83}$ recalculated due to (3.1).

8. With found functions $T_{j}, \omega_{j}$ and $\psi_{j}$ we solve numer- ${ }^{85}$ ically equation (3.5) to compute new position of the ${ }^{86}$ interface $\Gamma$ and new values of $v_{n}$.

9. Transition to the step 2 is carried out.

The proposed mathematical model and numerical ${ }^{90}$ algorithm allow one to describe a formation of gap in ${ }^{91}$ liquid layer. If condition $f<10^{-3}$ is fulfilled at some ${ }^{92}$ instant, it will be interpreted as a rupture of the lower ${ }^{93}$ fluid.

\section{Numerical investigations of fluid flow regimes 97} ing media. So far as the upper layer is taken as a ref-100 erence, and the characteristic velocity is chosen to bero1 equal to the velocity of viscous stresses relaxation, then the Reynolds number for gas layer $R_{1}$ is equal to 1 . The thicknesses of domains $\Omega_{1}$ and $\Omega_{2}$ at rest (at initial time $t=0$ ) are assumed to be the same, $h_{1}=$ $h_{2}=5 \cdot 10^{-3} \mathrm{~m}$. The characteristic temperature drop is taken equal to $T_{*}=10 \mathrm{~K}$, the length of the test section is $X=0.2 \mathrm{~m}$. In all cases, the size of the heater is the same $4 h_{1}=0.02 \mathrm{~m}$. The thermophysical properties of the fluids are given below in the order \{nitrogen (1), ethanol (2)\}, while the value of temperature coefficient of surface tension is specified only for ethanol: $\rho=\left\{1.25,0.79 \cdot 10^{3}\right\} \mathrm{kg} / \mathrm{m}^{3}, \nu=\left\{0.15 \cdot 10^{-4}\right.$ $\left.0.15 \cdot 10^{-5}\right\} \mathrm{m}^{2} / \mathrm{s}, \quad \beta=\left\{3.67 \cdot 10^{-3}, 0.108 \cdot 10^{-2}\right\} \mathrm{K}^{-1}$, $\chi=\left\{0.3 \cdot 10^{-4}, 0.89 \cdot 10^{-7}\right\} \mathrm{m}^{2} / \mathrm{s}, \quad \kappa=\{0.02717$, $0.1672\} \mathrm{W} /(\mathrm{m} \cdot \mathrm{K}), \quad \sigma_{T}=0.8 \cdot 10^{-4} \mathrm{~N} /(\mathrm{m} \cdot \mathrm{K})[23]$. All calculations are carried out with the following values of the defining dimensionless criteria: $\mathrm{Ca}=2 \cdot 10^{-3}$, $\mathrm{Ma}=112, \operatorname{Pr}_{1}=0.5, \operatorname{Pr}_{2}=16.8, \mathrm{Re}_{2}=10$. Values $\mathrm{G}=545, \mathrm{Gr}_{1}=200, \mathrm{Gr}_{2}=5751$ correspond to the terrestrial gravity $\left(g=g_{0}=9.81 \mathrm{~m} / \mathrm{s}^{2}\right)$. If microgravity conditions are considered then these parameters have the following magnitudes: $\mathrm{G}=5.45, \mathrm{Gr}_{1}=2$, $\mathrm{Gr}_{2}=57.51\left(g=g_{0} \cdot 10^{-2}\right)$.

We consider case of the commutated heating mode. In this regime the temperature of the heater increases abruptly from zero to a certain limit value at given instants in time and then the temperature drops to zero. Similar temperature regime simulates activating the heater, its switching to more intensive modes and the shutdown.

\subsection{Commutated heating from below}

We investigate the behavior of the system characteristics when one heater is arranged in the center of the substrate. The heater after switching on has a temperature $\theta^{s}(0)=0.1$, then the temperature is increased abruptly at fixed intervals: $\theta^{s}(10)=0.25, \theta^{s}(20)=0.5$, $\theta^{s}(30)=0.75, \theta^{s}(40)=1, \theta^{s}(50)=0$.

When a thermal source is switched on, we first observe the formation of convective cells generated by the action of mass forces. A hotter liquid rises up under the effect of buoyancy forces (Fig. 2(a)). Double-vortex flow pattern is formed in both liquid and gas layer. The heat from the thermal source is transferred into the liquid bulk by convective transport. Once the heat reaches the interface, surface forces come into action. The thermocapillary effect induces spreading the liquid along the interface and transferring heat from hot pole to the periphery (in domains with a lower temperature). It causes the formation of additional vortices and interface deformation. The transition from two-vortex flow pattern to quadruple-vortex structure occurs in both 
fluids (Fig. 2(b)). With each changeover of the heater ${ }_{54}$ its temperature is increased and the system responds 55 to a change in the thermal load with a certain lag time. 56 In the four-vortex regime under heater switching the ${ }_{57}$ thermocapillary deflection of the interface in the zone 58 of thermal exposure has two local dimples (Fig. 3(a)). 59 These concave meniscus are located above the areas of 60 ascending flows in each pair of vortices with opposite 61 circulation. Between these grooves there is an inflection 62 zone with a positive curvature (the surface is convex 63 outward).

Further increase of the thermal load leads to the ${ }^{65}$ hysteresis phenomena that appear by the oscillations ${ }^{66}$ of the interface and wave generation on the surface, ${ }^{67}$ and formation of the drifting vortices. Shapes of the ${ }^{68}$ interface with two menisci appear with the short-term ${ }^{69}$ lag period at every switching the heater. With time the ${ }^{70}$ surface oscillations damp, and form of the thermocap- ${ }^{71}$ illary deflection is stabilized (Fig. 3(c,e)). In commu- ${ }^{72}$ tated heating regime small vortices are generated near ${ }^{73}$ the hot spot in each layer. They are split out and travel ${ }^{74}$ to the side walls (Fig. 2(c,d)). Such oscillatory regimes ${ }^{75}$ arise only in the heater operation mode, when temper- ${ }^{76}$ ature $\theta^{s}$ is changed abruptly. If the temperature of the ${ }^{77}$ thermal sources does not change, then a stabilization of ${ }^{78}$ the secondary regime takes place. Upon that, a steady ${ }^{79}$ thermocapillary flexure of the interface with a negative ${ }^{80}$ curvature is formed in zone of thermal exposure.

The scenario of the system behavior is the same both in the terrestrial and microgravity conditions. Sub- 82 sequent transition from two-vortex pattern to quadruplevortex flow, and formation of vibrational modes that 83 are accompanied by appearance of traveling vortices 84 and oscillations of the interface under every changeover 85 of the heater are observed in low gravity also. But we 86 have elucidated essential differences for amplitudes of 87 the interface deformations and transverse size of ther- 88 mal patterns and planforms. At the same thermal expo- 89 sure the interface in the system being in weak force field 90 undergoes much greater deformations (compare ampli- 91 tudes of deformations for the interface in Figs. 3(a,c,e) ${ }_{92}$ and $(\mathrm{b}, \mathrm{d}, \mathrm{f}))$. Upon that, the delay time during which ${ }_{93}$ the system responds to a change in the temperature of ${ }_{94}$ heater is increased. It is explained by a weaker action 95 of the buoyancy forces, hence, a longer convective ris- 96 ing the hot liquid and heat transport to the surface. It 97 results in decelerated alteration of thermal field (com- 98 pare distribution of the temperature near the interface 99 in Figs. 3(a,c,e) and (b,d,f)). It should be noted alsoroo that under almost identical transversal size of thermo-101 capillary deflection the crosswise size of hot spot is sub-102 stantially smaller in microgravity in comparison with103 this under normal gravity. Emphasize that a further in-104 tensification of thermal load (increasing the heater temperature) leads to critical deformations and a rupture of the liquid layer at considered values of the gravity acceleration $\left(g=g_{0} \cdot 10^{2}\right)$.

When the thermal element is switched off the heat transfer in the system is supported only by the thermocapillary forces for some time (Fig. 4). In course of time a rest zone is formed in the central part of test section. At this, the transverse size of planforms in each layer and amplitude of thermocapillary deflection are decreased gradually (Fig. 5(a)). In the weak field of mass forces the system relaxes more slowly than in the terrestrial conditions. The process is accompanied by visible oscillations of the interface with a formation of two menisci (Fig. 5(b)) and longtime existence of vortices with two cores (Fig. 4(b)).

According to the results obtained we can talk about the instability of the equilibrium state of the two-layer system subjected by a local non-stationary heating from below. The instability is caused by the joint action of convective and thermocapillary mechanisms, and is evident as oscillatory regimes. It should be taken into account that in microgravity conditions significant thermal load and commutated heating can lead to a rupture of a liquid layer and formation of dry spot. Therefore, it should be possible to have alternative variants of operation mode for the heater to provide fail-safe functioning the system.

\subsection{Commutated heating from above}

One of the ways to avoid critical deformations of the interface in the two-layer system under microgravity conditions is to arrange the heater on the upper wall of the working section. In this case even under commutated heating mode, which is the most unfavourable regime of thermal exposure, the interface will be less sensitive to the thermal load (Figs. 6, 7). Presented results are obtained for the case when one heater is arranged in the center of the upper wall, and the thermal load is applied according to the rule: $\theta^{u p}(0)=0.1, \theta^{u p}(10)=0.25$, $\theta^{u p}(20)=0.5, \theta^{u p}(30)=0.75, \theta^{u p}(40)=1, \theta^{u p}(50)=$ 0 .

The deformation amplitudes are much smaller than those where system is heated from below. When the intensity of the thermal load is changed, no vibrational phenomena are observed in the system. In contrast to situations, when the heater is located on the substrate, we observe the inertial behavior of fluids, when system resists monotonically to changes in its state caused by local heating from above. The lack of the effect of the convective mechanism leads to a significant stabilization of the interface and the entire system. The heat 

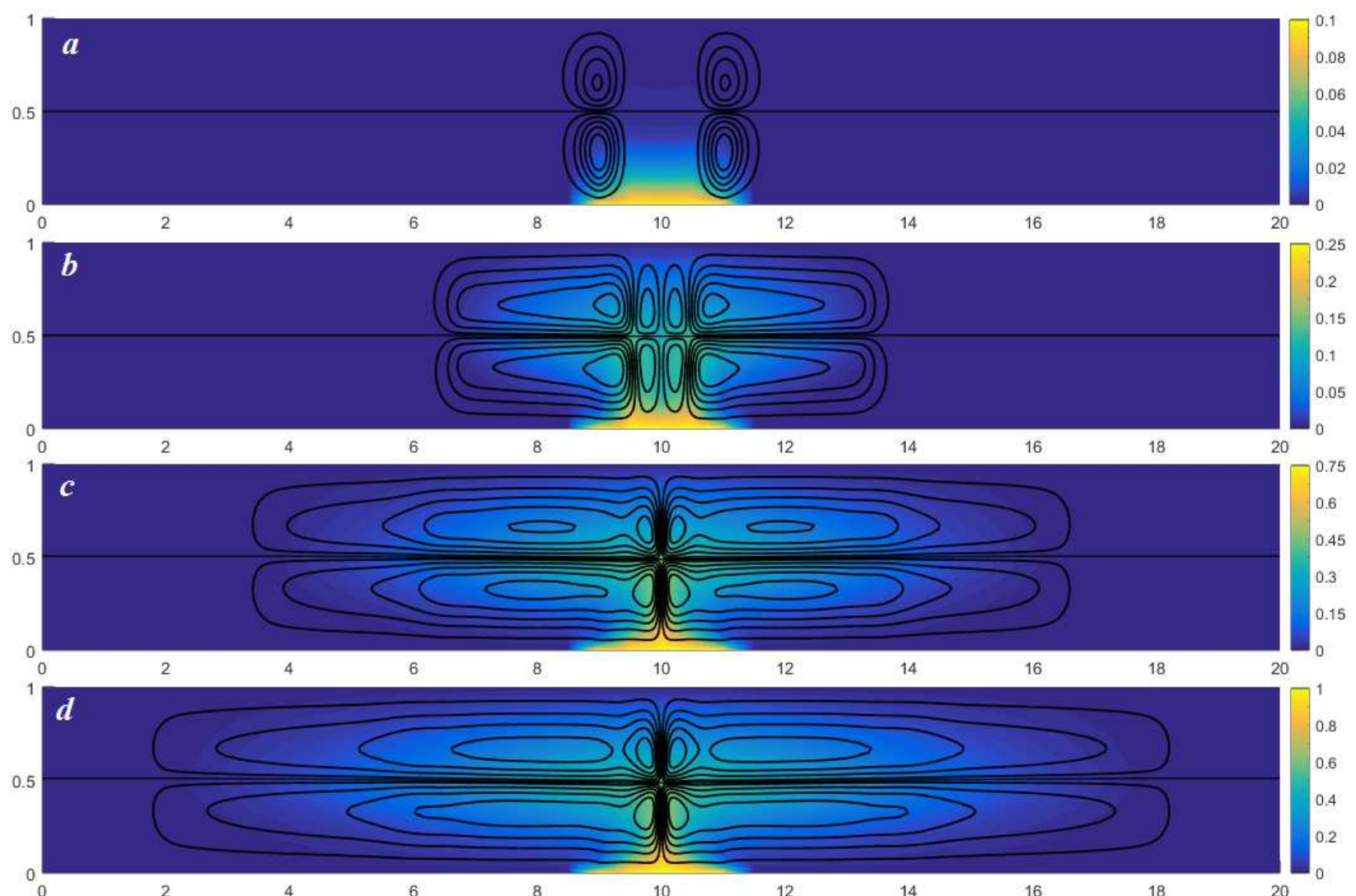

Fig. 2 Evolution of the thermal field and topological pattern of the flow in the system under commutated heating by a heater on substrate at $t=3 \mathrm{~s} \mathrm{(a),} t=16 \mathrm{~s}(\mathrm{~b}), t=33 \mathrm{~s}$ (c), $t=44 \mathrm{~s} \mathrm{(d).}$
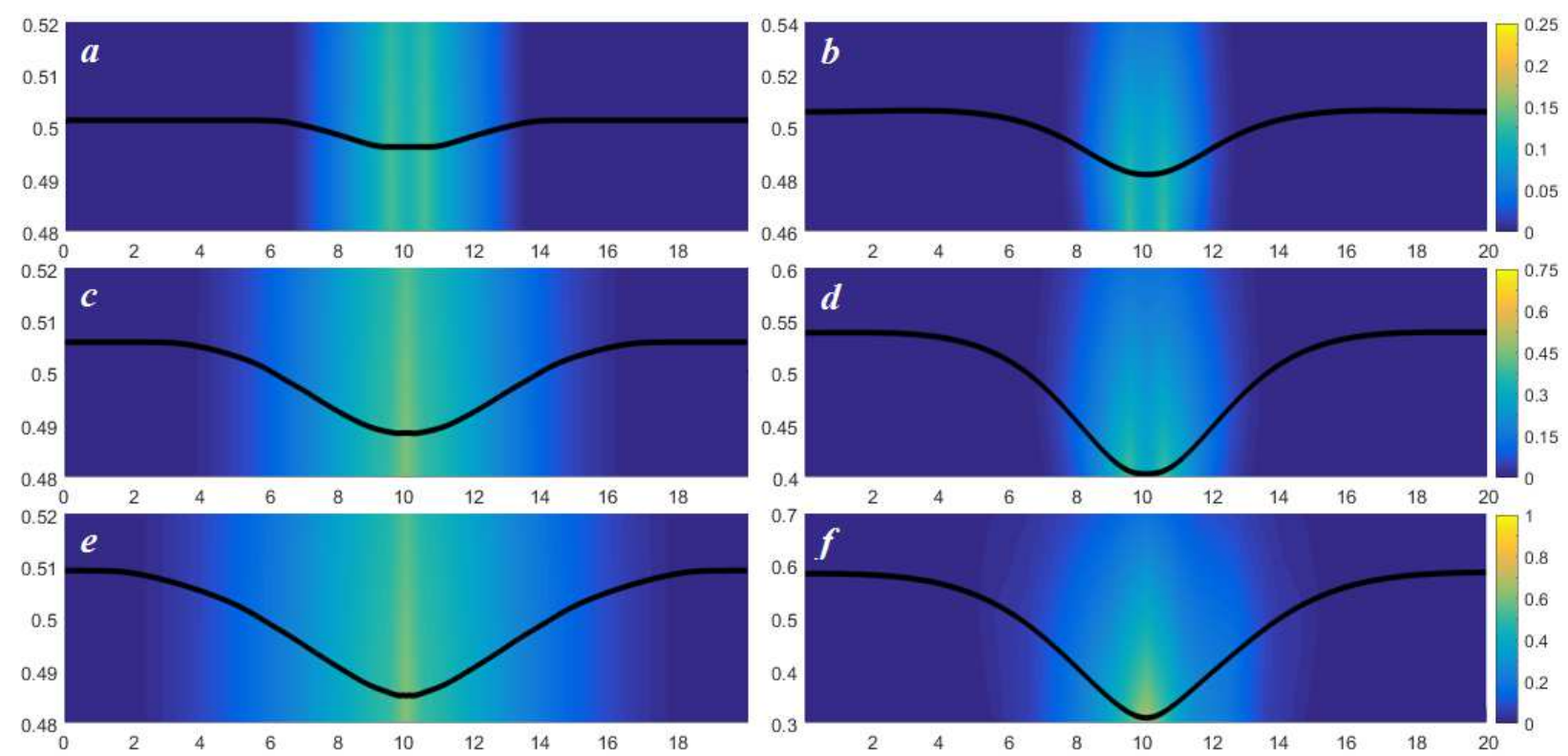

Fig. 3 The interface location and temperature near the surface under commutated heating by a heater on substrate at $t=16 \mathrm{~s}$ $(\mathrm{a}, \mathrm{b}), t=33 \mathrm{~s}(\mathrm{c}, \mathrm{d}), t=44 \mathrm{~s}(\mathrm{e}, \mathrm{f})$ in the terrestrial (a,c,e) and microgravity (b,d,f) conditions.

transfer from the heater to the interface is provided only 4 similar: a double-vortex flow is formed in each layer. by the thermal properties of the upper fluid (Fig. 6). 5 With an increase in the intensity of the thermal load Upon that, the flow structures in the liquid and gas are 6 produced by the heater, the size of the vortices does 


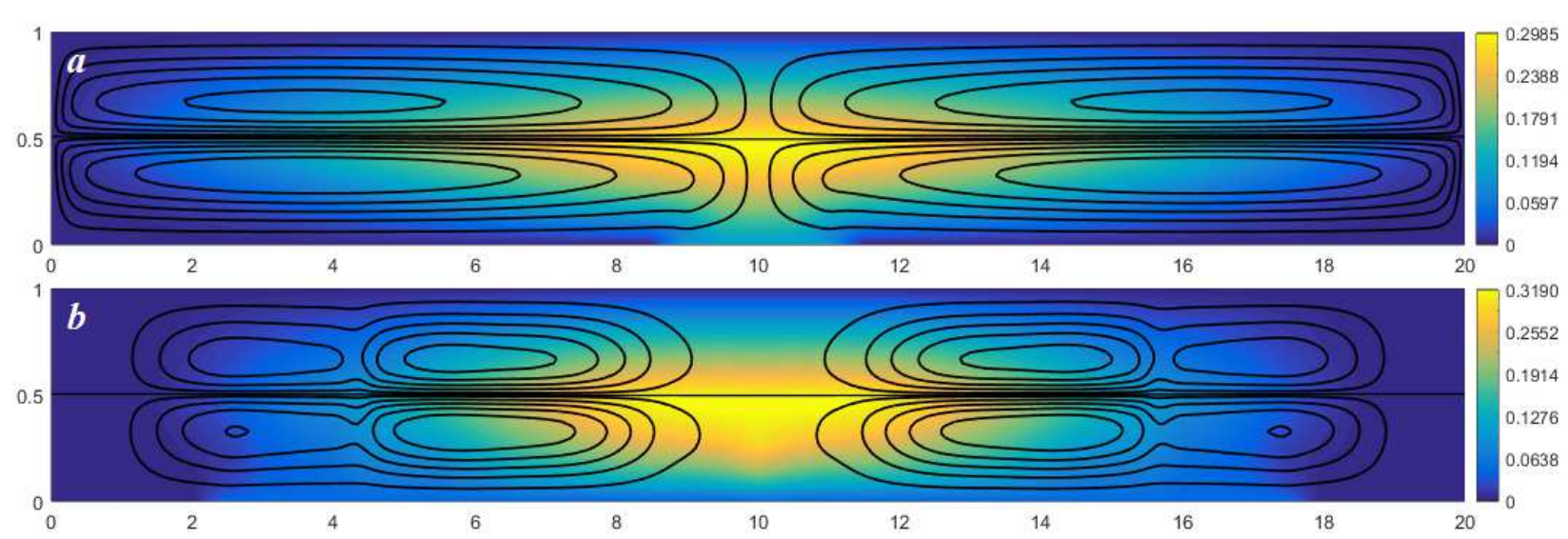

Fig. 4 The thermal field and topological pattern of the flow in the system 5 seconds after switching off the heater on substrate in the terrestrial (a) and microgravity (b) conditions.
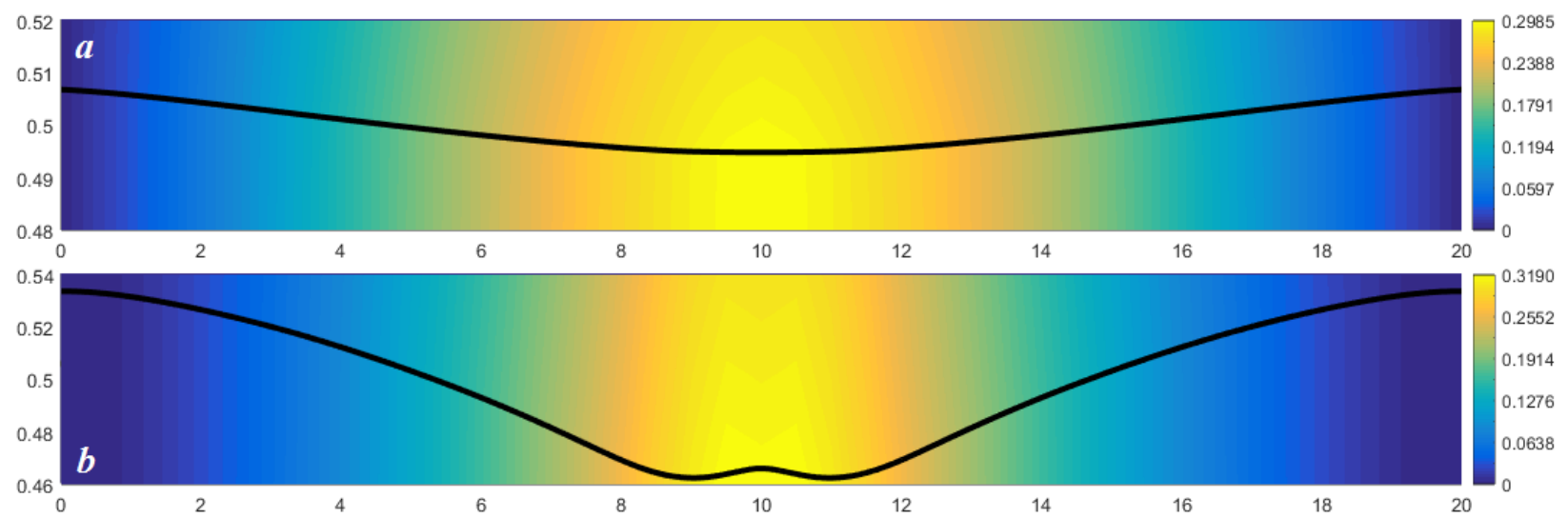

Fig. 5 The interface location and temperature near the surface 5 seconds after switching off the heater on substrate in the terrestrial (a) and microgravity (b) conditions.

not changed, and slight thermocapillary deflection is ${ }_{23}$ formed on the interface (Fig. 7).

Under normal gravity similar flow pattern is ob- 25 served in the system. A steady thermocapillary deflec- 26 tion is formed on the interface, and amplitude of the 27 flexure is much less than this in microgravity condi- 28 tions.

Thus, the loss of equilibrium stability for the considered two-layer system at a local commutated heating from above is accompanied by a formation of double- 30 vortex pattern both in the upper gas layer and in the lower liquid layer. Since the thermal conductivity of the ${ }_{31}$ gas is low, the heat from the thermal source to the in- 32 terface is transmitted poorly, and the movement in the 3 liquid layer is rather weak compared to the case where ${ }_{34}$ the heaters are located on the substrate. The liquid mo- 35 tion is induced by the action of Marangoni forces, which 36 cause thermocapillary spreading of the liquid along the 37 interface from the zones with higher temperature to the ${ }_{38}$ cold domains, and the subsequent vortex flow in the 39 bulk of liquid due to the properties of the medium con- 40 tinuity. Note, that at the initial stage of heating more ${ }_{41}$ intense movement in the gas phase is observed. It is explained by significantly different viscous properties of working fluids. It is the viscosity that characterizes the ability of media to resist gradual deformation of shear and/or tensile stresses. Over time, the intensity of flows in the layers is equalized due to the thermocapillary effect.

\section{Conclusions}

The problem of convection onset in a two-layer system subjected by a local heating has been considered. The mathematical model based on the Oberbeck-Boussinesq convection equations was used to describe the motion and heat transfer in system with internal interface under a local thermal exposure. Formulation of all the boundary conditions, including relations on the interface, in explicit form is presented. The suggested approach allows one to take fully into account the influence of the vertical velocity component and the contribution of convective summands to the formation of the 

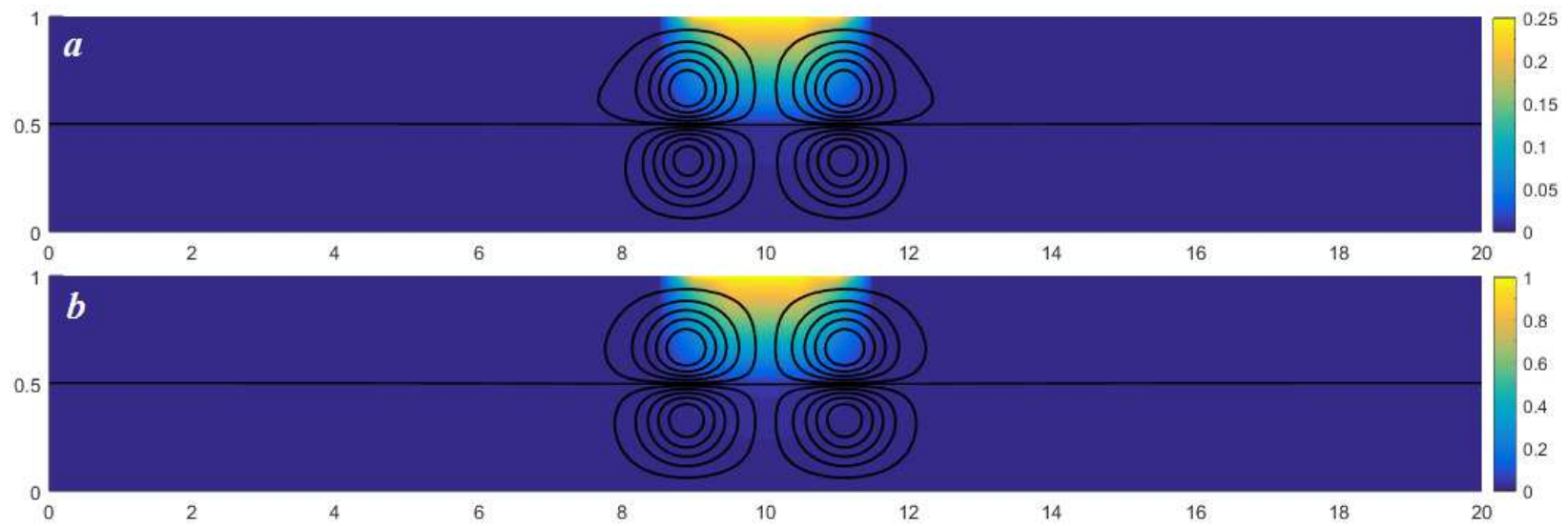

Fig. 6 Evolution of the thermal field and topological pattern of the flow in the system subjected to commutated heating from above in microgravity conditions at $t=16 \mathrm{~s} \mathrm{(a),} t=44 \mathrm{~s}(\mathrm{~b})$.
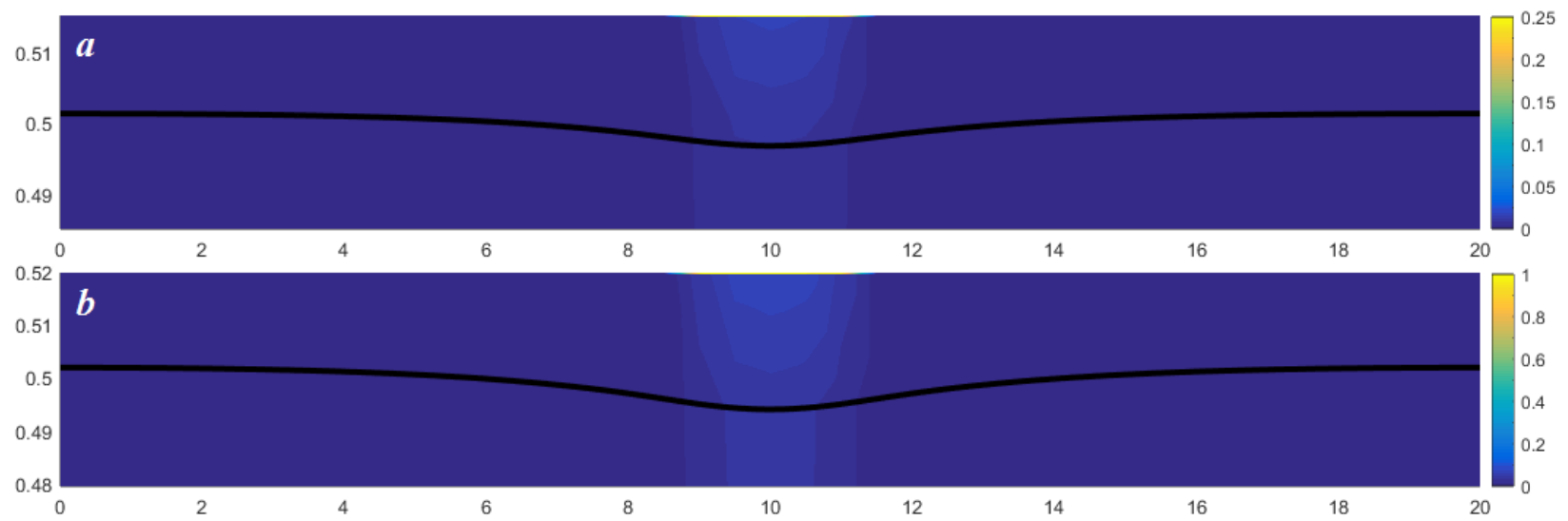

Fig. 7 The interface location and temperature near the surface under commutated heating from above in microgravity at $t=16 \mathrm{~s}(\mathrm{a}), t=44 \mathrm{~s}(\mathrm{~b})$.

observed flow regimes, both in terms of hydrodynamic 22 and thermal aspects.

The appearance of motions with different planforms ${ }^{24}$ caused by the combined action of convective and ther- ${ }^{25}$ mocapillary mechanisms, and various scenarios of the ${ }^{26}$ surface behavior under low and normal gravity have ${ }^{27}$ been described. With the help of numerical simulation ${ }^{28}$ it was shown that occurrence of hysteresis phenomena ${ }^{29}$ may be resulted in non-stationary thermal load with discontinuous changes of heater temperature. There is ${ }^{30}$ a certain lag time during which the system responds ${ }_{32}^{31}$ to a change in the intensity of thermal exposure. The ${ }_{33}$ delay period depends on the intensity of gravity field, in a weak force field the lag time is increased. Furthermore, under microgravity non-uniform heating can lead ${ }^{34}$ to a rupture of liquid layer due to the critical deformations generated by the thermocapillary effect. Action ${ }_{36}$ of the Marangoni forces causes a significant growth of 37 the tangential velocity along the interface, which is the ${ }_{39}^{38}$ main reason of the appearance of the critical flexure of ${ }_{40}^{39}$ the surface between fluids. In deactivating the thermal 41 load in the weak field of mass forces the system relaxes more slowly than in the terrestrial conditions. Arrangement of the heater from above allows one to reduce the convective mechanism action. It results in an essential stabilization of the interface and the inertial behavior of the system instead of hysteresis.

Conflict of Interest: The authors declare that they have no conflict of interest.

Acknowledgements The authors are very grateful to Dr A. S. Ovcharova for helpful discussions and adaptation of the code for solving of the non-stationary problems with interfaces.

\section{References}

1. Andreev V.K., Gaponenko Yu.A., Goncharova O.N., Pukhnachov V.V., Mathematical models of convection (de Gruyter Studies in Mathematical Physics), 430. De Gruyter, Berlin, Boston, 2012

2. Gershuni G.Z., Zhukhovitskii E.M., Convective Stability of an Incompressible Fluid, 392. Nauka, Moscow, 1972 [in Russian] 
3. Colinet P., Legros J.C., Velarde M.G., Nonlinear Dynamics of Surface-Tension-Driven Instabilities, 512. WileyVCH, Berlin, 2001.

4. Nepomnyashchy A.A., Velarde M.G., Colinet P., Interfacial phenomena and convection, 360. Chapman \& Hall/CRC, Boca Raton, 2002

5. Nepomnyashchy A.A., Simanovskii I.B., Legros J.-C., Interfacial convection in multilayer systems, 498. Springer, New York, 2015

6. Margerit J., Colinet P., Lebon G., Iorio C.S., Legros J.C., Interfacial nonequilibrium and Benard-Marangoni instability of a liquid-vapor system, Phys. Rev., E 68, 041601-1041601-14 (2003)

7. Bekezhanova V.B, Goncharova O.N., Problems of the Evaporative Convection (Review), Fluid Dyn., 53(1), 69$102(2018)$

8. Napolitano L.G., Thermodynamics and dynamics of surface phases, Acta Astronautica, 6(9), 1093-1012 (1979)

9. Pukhnachov V.V., Viscous fluid flow with free boundaries, Novosibirsk State University, Novosibirsk, 1989 [in Russian].

10. Doerfler W., Goncharova O., Kroener D., Fluid flow with dynamic contact angle: numerical simulation, ZAMM, 82 (3) $167-176$ (2002)

11. Goncharova O., Zakurdaeva A., Legros J.-C., Investigation of behavior of the dynamic contact angle on the basis of the Oberbeck-Boussinesq approximation of the NavierStokes equations, MATEC Web of Conferences, 84, 00014 (2016)

12. Bekezhanova V.B., Ovcharova A.S., Thermocapillary convection in a liquid layer induced by local heating, Journal of Fluid Mechanics (submitted for publication) (2018)

13. Ovcharova A., Stankus N., A deformation and a break of hanging thin film under microgravity conditions, Fluid Dynamics \& Materials Processing, 3(4) 349-356 (2007)

14. Ovcharova A.S., Rupture of liquid film, placed over deep fluid, under action of thermal load, Int. J. Heat and Mass Transfer, 78, 294-301 (2014)

15. Iorio C.S., Kabov O.A., Legros J.-C., Thermal Patterns in evaporating liquid, Microgravity Science and Technology, XIX(3/4), 27-29 (2007)

16. Lyulin Y.V., Kabov O.A., Evaporative convection in a horizontal liquid layer under shear-stress gas flow, Int. J. Heat Mass Transfer, 70, 599-609 (2014)

17. Lyulin Y.V., Spesivtsev S.E., Marchuk I.V., Kabov O.A., Study of the dynamics of rupture of thin liquid layers under point heating and droplet cluster formation, Thermophysics and Aeromechanics, 24(6), 977-980 (2017) [in Russian]

18. Barakhovskaya E., Marchuk I.V., Numerical modeling of thermocapillary deformation and film breakdown in a locally heated thin horizontal volatile liquid layer, MATEC Web of Conferences, 72, 01007 (2016)

19. Klyuev D.S., Fliagin V.M., Al-Muzaiqer M., Ivanova N.A., Laser-actuated optofluidic diaphragm capable of optical signal tracking, Applied Physics Letters (accepted for publication) (2018)

20. Roache P.J., Computational Fluid Dynamics, 446. Hermosa Publishers, Albuquerque, NM, USA, 1976

21. Douglas jr. J., Gunn J.E., A general formulation of alternating direction methods, I: Parabolic and hyperbolic problems, Numer. Math., 6, 428-453 (1964)

22. Yanenko N.N., The method of fractional steps: the solution of problems of mathematical physics of several variables, 160. Springer Verl., Berlin, Heidelberg, New York, 1971

23. Weast C.R.C., Handbook of Chemistry and Physics. CRC Press Inc., Boca Raton, Florida, 1979 